\title{
Die ersten Ergebnisse der Beobachtungen mit dem Repsoldschen Heliometer der v. Kuffnerschen Sternwarte. Von L. de Ball.
}

Das Heliometer der v. Kuffnerschen Sternwarte ist gegenwärtig nur durch die von den Herren Repsold in Band IV unserer Publikationen gegebene Beschreibung desselben bekannt. Daß aber das schöne Instrument auch in Bezug auf seine Leistungsfähigkeit vollauf den Erwartungen entspricht, zu denen der Name der Künstler, welche es geschaffen haben, die Astronomen berechtigte, soll durch die nachfolgende Mitteilung über die Resultate der Bearbeitung einer 7-jährigen, in sich geschlossenen Beobachtungsreihe gezeigt werden.

Bei der Ausführung der Beobachtungen habe ich die Regeln befolgt, welche sich bei den von anderen Heliometerbeobachtern angestellten Untersuchungen als zweckmäßig bewährt haben und heutzutage als allgemein bekannt angesehen werden dürfen. Ich habe aber außer diesen noch eine Regel berücksichtigt, welche erst weiter unten angegeben werden soll und die möglicherweise nicht ohne Bedeutung für die Elimination gewisser systematischer Fehler ist. Was die Reduktion der Beobachtungen betrifft, so ist zunächst einer Vereinfachung des vielfach angewandten Rechnungsverfahrens Erwähnung zu tun. Bisher begann man gewöhnlich damit, die bei den Fokusbestimmungen erhaltenen $A b$ lesungen der Okularskala durch die Formel auszugleichen

$$
F=x+y t
$$

wo $F$ die der an einem Abende beobachteten Fokalstellung des Fadenkreuzes entsprechende Ablesung der Okularskala, $t$ die zugehörige Temperatur, und $x$ und $y$ zwei zu bestimmende Konstanten bedeuten. Mit Hilfe der wahrscheinlichsten Werte von $x$ und $y$ ließ sich dann eine 'Tafel berechnen, aus der man für jede beobachtete Temperatur den Wert von $F$ entnehmen konnte. Der für die Temperatur $t$ aus der Tafel entnonmene Wert von $F$ möge mit $N$, und die dieser Ablesung der Okularskala entsprechende Stellung des Okulars als die Normalokularstellung für die Temperatur $t$ bezeichnet werden. Hatte man nun bei der Temperatur $t$ eine Distan $z$ beobachtet, und war die der Stellung des Okulars zur Zeit der Beobachtung entsprechende Ablesung der Okularskala gleich $O$, so wurde die Distanz zunächst auf die Normalokularstellung reduziert, d. h. es wurde an die gemessene, in Einheiten eines Intervalls der Heliometerskalen ausgedrückte Distanz $d$ eine Korrektion $(C)$ von der Form

$$
C=h(d / \mathrm{r} \circ 0)(N-O)
$$

angebracht, wo $h$ die für $d=$ ı 00 Intervalle der Heliometerskalen und $N-O=$ I Intervall der Okularskala gültige Korrektion bedeutet. Diese reduzierten Distanzen bildeten die Grundlagen der weiteren Rechnung.

Das eben skizzierte Verfahren hatte auch ich angewandt, als ich aus meinen Beobachtungen der großen Distanzen im Pol- bezw. Cygnusbogen zunächst nur näherungsweise den Winkelwert eines Intervalls der Heliometerskalen bestimmen wollte. Erst nachdem dieses Ziel erreicht war, kam ich zu der Überzeugung, daß die ganze vorhin erwähnte Rechnung unnötig sei und man besser daran täte, alle Distanzen auf ein und dieselbe Okularstellung zu reduzieren. Den letzteren Weg habe ich bei der definitiven Berechnung meiner Beobachtungen eingeschlagen und damit den großen
Zeitgewinn, den man bei Anwendung des neuen Verfahrens erzielt, aus eigener Erfahrung feststellen können. Die Reduktion auf eine konstante Okularstellung (besser gesagt: auf eine konstante Ablesung der Okularskala) wird namentlich dann sehr einfach, wenn man vor Beginn der Beobachtungen dafür sorgt, daß die Ablesung der Okularskala auf ein der Fokalstellung möglichst nahes volles Zehntel endigt. Bei meinen Beobachtungen z. B. kommen nur die Ablesungen I 9.70 , I $9.80, \cdots, 20.30,20.40$ vor; reduziert wurden alle gemessenen Distanzen auf die Ablesung 20.00. Diese Arbeit war in wenigen Minuten erledigt.

Um die Reduktion der gemessenen Distanzen auf die Ablesung 20.00 der Okularskala ausführen zu können, war die Bestimmung der in (2) mit $h$ bezeichneten Konstante erforderlich. Hierzu dienten die an demselben Abende bei 2 oder 3 verschiedenen Ablesungen der Okularskala angestellten Messungen der Distanz der Endsterne ( $a$ und $c$ ) des aus dem Auwersschen Bericht über die Venusdurchgänge bekannten Polbogens. Die Örter dieser Sterne sind:

$$
\begin{array}{llll} 
& \alpha(\mathrm{I} 900) & \delta(\mathrm{Ig00}) \\
a & \mathrm{II}^{\mathrm{h}} \mathbf{2}^{\mathrm{n}} \cdot 5 & +86^{\circ} \mathrm{II}^{\prime} \\
e & \text { I } 2 & 59.7 & +8625
\end{array}
$$

Daß, nachdem die Distanz beispielsweise für die Ablesungen $19.40,20.00,20.40$ der Okularskala gemessen war, die Messungen in der umgekehrten Reihenfolge wiederholt wurden, ist eigentlich selbstverständlich, möge aber doch noch ausdrücklich erwähnt werden. Zur Ausgleichung der an einem Abende bei verschiedenen Okularstellungen erhaltenen Werte der Distanz wurde die Formel angewandt

$$
d=d_{0}+h(d / \mathrm{ro0})\left(O-O_{0}\right)
$$

wo $d$ die der Ablesung $O$ der Okularskala entsprechende gemessene Distanz, und $d_{0}$ die für eine fest angenommene Ablesung $O_{0}$ gültige Distanz bedeutet. Die aus diesen Gleichungen sich ergebenden Werte von $d_{0}$ sind für das Folgende ohne Interesse. Es genügt sonit, hierunter nur die für $h$ gefundenen Werte, ausgedrückt in Teilen eines Intervalls der Heliometerskalen $\left({ }_{1} \mathrm{~S}\right)$, mitzuteilen; dabei bedeutet $K$ die durch. das am Objektivkopfe befindliche Thermometer angezeigte Temperatur.

$$
\begin{aligned}
& \begin{array}{cccc} 
& h & h & \text { Gewicht } \\
\text { I } 902 \mathrm{Mai} 30 & +17.2 & 0.0264 & 0.125
\end{array} \\
& \begin{array}{lllll}
\text { Juni } 2+22.3 & 0.0228 & 0.125
\end{array} \\
& 4+21.0 \quad 0.03150 .50 \\
& 9+12.80 .0300 \quad 0.50 \\
& \text { Aug. } 16+17.1 \quad 0.0280 \quad 0.50 \\
& \text { Sept. } 5+21.8 \quad 0.0304 \quad 0.50 \\
& \text { I } 903 \mathrm{Juli} \text { I } 2+20.6 \quad 0.03220 .50 \\
& \text { I } 5+20.0 \quad 0.0300 \quad 0.50 \\
& \text { I } 906 \text { Aug. } 29+\text { I } 3.7 \quad 0.0307 \quad 0.3^{2} \\
& 31+17.4 \quad 0.0321 \quad 0.32 \\
& \begin{array}{llll}
\text { Sept. } I+18.5 & 0.0313 & 0.5^{\circ}
\end{array} \\
& \text { Nov. } 15+1.0 \quad 0.0333 \quad 0.32 \\
& 20+6.20 .0327 \quad 0.32
\end{aligned}
$$

Hieraus folgt als Mittelwert $h=0.0306, \mathrm{~m} . \mathrm{F} . \pm 0 \$ 0006$. Für die Reduktion der bei der Ablesung $O$ der Okularskala 
gemessenen Distanzen $(d)$ auf die Ablesung 20.00 wurde demnach die Formel benutzt

$$
d_{0}=d+0.0306(d / 100)(20.00-0) \text {. }
$$

Nachdem diese Formel gefunden war, konnte die Berechnung aller Beobachtungen der Distanz ae des Polbogens (mit Ausschluß der für die vorige Untersuchung nötigen, in größerer Entfernung vom Fokus angestellten), sowie der Distanzen $a d, c f, b e$ des Cygnusbogens in Angriff genommen werden; mit Hilfe der hierbei gewonnenen Resultate und der Formel für $d_{0}$ läßt sich später die Reduktion meiner Parallaxenbeobachtungen ausführen. $\mathrm{Da}$ es für die folgenden Untersuchungen nötig war, die Örter und Eigenbewegungen der beobachteten Sterne zu kennen, so bemerke ich, daß mir außer den von Auwers benutzten Sternpositionen auch noch neuere, in Pulkowo, Greenwich, München, Mt. Hamilton und Washington erhaltene Ortsbestimmungen $2 u$ Gebote standen. Eine nähere Mitteilung über die Bearbeitung des ganzen Materials würde viel Raum beanspruchen, und soll deshalb erst gelegentlich der Veröffentlichung der Ergebnisse meiner Heliometerbeobachtungen in den Publikationen unserer Sternwarte erfolgen; hier seien nur noch die genäherten Örter der Sterne des Cygnusbogens angeführt:

$\begin{array}{lccc} & \Delta_{0} & b_{1} & \text { Gewicht } \\ \text { Polbogen } & 196.83 & +0.0027 & 85 \\ \text { Cygnus } d a & 186.97 & +0.0023 & 153 \\ \Rightarrow \quad f c & 183.34 & +0.0023 & 101 \\ " \quad e b & 172.29 & +0.0021 & 123\end{array}$

Wenngleich die Differenz zwischen dem aus den Beobachtungen der Distanz Cygnus $d a$ folgenden Werte von $c_{1}$ und den drei anderen nicht viel größer ist, als man auf Grund der Gewichte und der unten angefuihrten mittleren Fehler einer Distanz erwarten kann, so habe ich doch untersuchen wollen, ob sich dieselbe durch eine Parallaxendifferenz der Sterne $d$ und $a$ erklären lassen könne. Bedeutet $\pi$ die Parallaxe von $d$, und $\pi_{i}$ die Parallaxe von $a$, denkt man sich ferner $\pi$ und $\pi_{i}$ in Teilen eines Skalenintervalls ausgedriickt, so sind die für $b_{1}$ und $c_{1}$ erhaltenen Ausdrücke:

$$
\begin{array}{ll}
\text { (Cygnus da) } \quad b_{1}=+0.0023-0.003\left(\pi-\pi_{i}\right) \\
c_{1}=-0.0003^{2}+0.032\left(\pi-\pi_{i}\right) .
\end{array}
$$

Für $\pi-\pi_{i}=+0$ "०5 $=+0$ o० 0 r 45 , beispielsweise, würde $c_{1}=-0.00027$ folgen, also ein Wert, der besser mit den übrigen zusammenstimmt. Nach den bei der Reduktion anderer Heliometerbeobachtungen (Peter, Schur) gemachten Erfahrungen ist es indessen wenig wahrscheinlich, daß es sich im vorliegenden Falle um die Wirkung einer Parallaxendifferenz handelt.

Auf Grund der vorigen Resultate habe ich nun

$$
\Delta_{0}=\Delta^{\prime}+0.0023\left(\Delta_{0} / 100\right)(E-1904.0)-
$$
- 0.000205 $\left(\Delta_{0} / 100\right) K-0.00019\left(\Delta_{0} /\right.$ I00) $(E-\text { I } 904.0)^{2}$ angenommen und diese Formel dazu benutzt, um aus den für die vier Sternpaare: $a e$ des Polbogens, $d a$, bezw. $f c$, bezw. $e b$ des Cygnusbogens erhaltenen $\Delta^{\prime}$ die Werte von $\Delta_{0}$ abzuleiten. Hier ist jetzt noch folgendes zu erwähnen. Bei der Beobachtung habe ich mich eines Okularprismas bedient. Bekanntlich kann man mit Hitfe desselben bewirken,
M. Äq. 1900 .

$d 20^{\mathrm{h}} 45^{\mathrm{m}} \cdot 7+5^{1^{\circ}} 32^{\prime} f 20^{\mathrm{h}} 43^{\mathrm{m}} \cdot 5+52^{\circ} 3^{8^{\prime}}$ e $20^{\mathrm{h}} 44^{\mathrm{m}} \cdot 9+52^{\circ} 3^{\prime}$

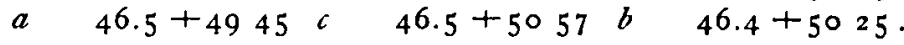
Die beobachteten Distanzen .wurden nun wegen der Teilungsfehler der Skalen und des Ganges der Schraube korrigiert, außerdem auf die Ablesung 20.00 der Okularskala reduziert, ferner von der Refraktion und Aberration befreit, und endlich mit Hilfe der aus den vorhin erwähnten Sternpositionen abgeleiteten Eigenbewegungen auf die Epoche 1904.0 gebracht; die so erhaltenen Distanzen mögen mit $\Delta^{\prime}$ bezeichnet werden. Mit Rücksicht darauf, daß die frühere vorläufige Bestimmung des Skalenwertes gezeigt hatte, daß letzterer nicht nur von der Temperatur abhänge, sondern sich auch nahezu proportional der Zeit ändere, habe ich den zur Ausgleichung der $\Delta^{\prime}$ dienenden Bedingungsgleichungen die Form gegeben

$$
\begin{aligned}
\Delta_{0}=\Delta^{\prime} & +b_{1}\left(\Delta_{0} / \text { roo }\right)(E-1904.0)+c_{1}\left(\Delta_{0} / \text { 100 }\right) K+ \\
& +d_{1}\left(\Delta_{0} / \text { 100 }\right)(E-1904.0)^{2}
\end{aligned}
$$

dabei bedeutet $E$ die Epoche der Beobachtung, und $\Delta_{0}$ die für die Ablesung des Objektivthermometers $K=0^{\circ}$ und die Epoche I 904.0 gültige Distanz. Aus diesen Gleichungen ergaben sich die folgenden Werte der Koeffizienten $b_{1}, c_{1}$ und $d_{1}$, ausgedrückt in Teilen eines Skalenintervalls:

$\begin{array}{crrc}c_{1} & \text { Gewicht } & d_{1} & \text { Gewicht } \\ -0.00020 & \text { I I973 } & -0.00027 & 375 \\ -0.00032 & 5548 & -0.00034 & 173 \\ -0.00020 & 8003 & -0.00016 & 369 \\ -0.00017 & 6721 & -0.00004 & 386\end{array}$

daß das Durchschwingen der Bilder in allen Lagen des Fernrohrs in einer und derselben Richtung erfolgt, und zwar wählt der eine Beobachter die horizontale, der andere die vertikale Richtung. Ich habe beide Richtungen angewandt. Wenn ich also in der einen Lage des Objektivkopfes die Bilder horizontal hatte schwingen lassen, so ließ ich für die Beobachtung in der um $180^{\circ}$ gedrehten Lage des Objektivkopfes die Bilder vertikal schwingen. Es blieb demnach $z u$ untersuchen, ob die Schwingungsrichtung einen Einfluß auf die Messungen hatte. Wenn nun $V$ den aus den Messungen bei vertikaler, und $H$ den aus den Messungen bei horizontaler Schwingungsrichtung folgenden Wert von $\Delta_{0}$ bedeutet, so sind die für die Differenz $V-H$ erhaltenen Mittelwerte:

$$
\begin{aligned}
& V-H \quad \text { m. F. } \\
& \text { Polbogen - }- \text { S.0028 } \pm 0 \text {.0010 } \\
& \text { Cygnus } d a-0.0012 \pm 0.0012 \\
& \nu f i-0.0023 \pm 0.0013 \\
& \nabla c b-0.0025 \pm 0.0013
\end{aligned}
$$

Die Existenz eines kleinen systematischen Unterschiedes zwischen den bei vertikaler und horizontaler Schwingungsrichtung gemessenen Distanzen scheint also ziemlich sicher zu sein.

Bildet man für jeden Bogen das Mittel aus allen $\Delta_{0}$, so erhält man die hierunter mit $M$ bezeichneten Werte. Zur Seite derselben findet man die aus den angenommenen Sternörtern berechneten, für die Epoche r904.0 gültigen Distanzen $M^{\prime \prime}$ mitgeteilt. Mit Hilfe von $M$ und $M^{\prime \prime}$ ergibt sich der in Bogensekunden ausgedrückte Wert eines Skalenintervalls $\left(\mathrm{r}^{\mathrm{S}}\right)$. 


\begin{tabular}{ccccc} 
& $M$ & $M^{\prime \prime}$ & \multicolumn{1}{c}{${ }^{\prime} \mathrm{S}$} & $M^{\prime \prime}-D$ \\
Polbogen & I96S8322 & 6780.47 & $34^{\prime \prime} 4480$ & -0.20 \\
Cygnus $d a$ & 186.9680 & 6440.97 & .4496 & +0.11 \\
$\Rightarrow \quad f c$ & 183.3436 & 6315.96 & .4487 & -0.05 \\
$\Rightarrow \quad e b$ & 172.2914 & 5935.48 & .4503 & +0.22
\end{tabular}

Hiernach kann man bis auf weiteres annehmen

$$
{ }_{\mathrm{I}} \mathrm{S}=34.449 \text {. }
$$

Die mit Hilfe dieses Wertes und der Werte von $M$ berechneten Distanzen $(D)$ unterscheiden sich von den $M^{n}$ um die in der letzten Kolumne angeführten Quantitäten.

Es sollen jetzt noch die Differenzen zwischen den für jeden Beobachtungstag erhaltenen $\Delta_{0}$ und den vorhin mit $M$

\section{Polbogen}

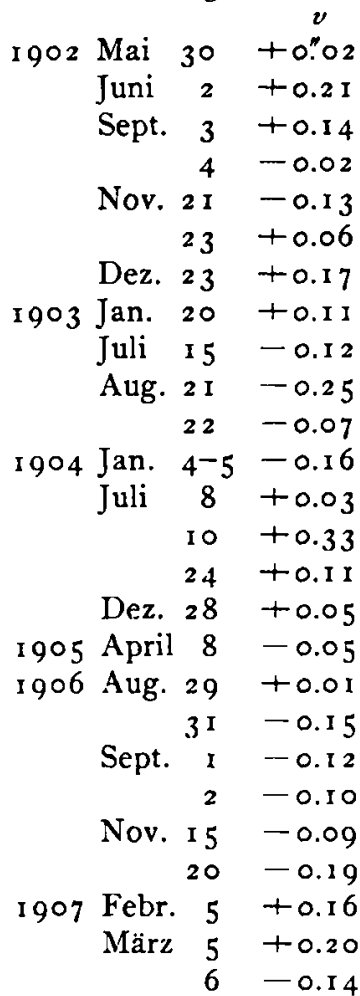

Cygnus $d a$

$$
\begin{aligned}
& \text { Igo I Okt. I +o". I } 3 \\
& 4+0.08 \\
& \text { Nov. } 13-0.23 \\
& \text { Dez. } 13+0.08 \\
& 1902 \text { März I } 3-0.19 \\
& \text { Juni } 29+0.03 \\
& 30+0.03 \\
& \text { Nov. } 2 \text { I }-0.08
\end{aligned}
$$

I 903 Aug. 2 I-2 $2+0.1$ I

I 904 Sept. I $3-0.01$

1906 Febr. $17-0.16$

April $4+0.01$

Juni I $9+0.18$

Juli $5+0.02$ bezeichneten Mittelwerten der $\Delta_{0}$ angeführt werden. Zur besseren Orientierung des Lesers gebe ich diese Differenzen $\left(v=\Delta_{0}-M\right)$ nicht in Teilen eines Skalenintervalls, sondern in Teilen einer Bogensekunde ausgedrückt an. - Am 4. und 5. Januar 1904 wurde der Polbogen jedesmal nur in einer Lage des Objektivkopfes gemessen, doch war diese Lage für den einen Tag die entgegengesetzte wie für den anderen; ferner war die Schwingungsrichtung der Bilder am ersten Tage horizontal, am zweiten vertikal. Mit Rücksicht hierauf habe ich für das Folgende den Mittelwert der beiden $\Delta_{0}$ benutzt. Eine analoge Bemerkung gilt auch für die am 2 I. und 22 . August $19 \circ 3$ angestellten Beobachtungen der Distanz Cygnus $d a$.

\begin{tabular}{rrr}
\multicolumn{3}{c}{ Cygnus $f c$} \\
I90 I Okt. 3 & -0.04 \\
& Nov. I 7 & +0.06 \\
1902 & Nov. 23 & +0.05 \\
I903 Jan. 20 & +0.02 \\
Aug. 24 & -0.12 \\
Sept. 30 & 0.00 \\
Okt. 8 & +0.01 \\
I904 Sept. I 3 & -0.01 \\
I905 Jan. & 2 & +0.07 \\
& & +0.01 \\
& Aug. 10 & +0.01 \\
Dez. 17 & -0.01 \\
1906 Sept. 3 & +0.04 \\
& 4 & +0.03 \\
I907 Jan. 24 & -0.05 \\
& 27 & -0.08
\end{tabular}

Die überraschend hohe Genauigkeit, welche sich dem 7 -zölligen Heliometern ergeben hat, so drängt sich unwillvorstehenden zufolge mit unserem Heliometer erreichen läßt, hat sich auch bei einer vorläufigen Reduktion der zur Bestimmung der Parallaxen von drei Sternen angestellten $\mathrm{Be}$ obachtungen gezeigt. Bedenkt man nun, daß Gill mit dem 7-zölligen Heliometer der Kap-Sternwarte eine beträchtlich größere Genauigkeit seiner Parallaxenbeobachtungen erzielte als mit dem früher von ihm benutzten 4-zölligen, und daß der aus den mit unserem 8-zölligen Heliometer angestellten Messungen der obigen 4 Distanzen im Pol- und Cygnusbogen folgende mittlere Fehler einer Beobachtung wieder merklich kleiner ist, als er sich für gleich große Distanzen bei 6- und

kürlich der Wunsch auf, daß bald ein 9- oder gar ro-zölliges Heliometer konstruiert werden möge. Das Heliometer steht leider in dem üblen Rufe, daß es sehr schwierig zu handhaben sei und ungewöhnliche Anforderungen an den Beobachter stelle; für die modernen Heliometer trifft das aber keineswegs zu. Wer mit einem Refraktor umgehen kann, wird gar keine Schwierigkeiten haben, an einem Heliometer zu beobachten. Hauptbedingung für den Erfolg ist, daß man die Messungen mit der nötigen Ruhe ausführt, also von vornherein auf jede "Fixigkeit" Verzicht leistet.

Wien XVI, I9I3 Jan. I I.

L. de Ball.

Allgemeine Schätzungen betreffend den Kometen 1911 c (Brooks). Von Dr. A. Kaiser in Prag. (Ergänzung zu Nr. 4623 der A. N.)

IgI I Sept. 26. $8^{1 / 2^{\mathrm{h}}} \mathrm{m}$. Z. Prag. Schweif ist schön Kometensuchergesichtsfeldes, also gegen $5^{\circ}$. Er ist gerade ausgebildet und erstreckt sich über $3 / 4$ Durchmesser des und schmal und nach OSO gerichtet. Die Haube reicht über 